\title{
Tracheobronchial Foreign Body Aspiration Demonstrating Serial Bronchopulmonary Changes on Computed Tomography
}

\author{
Hidehiro Watanabe ${ }^{1,2,}$; Tomonori Uruma $^{1,2}$; Gen Tazaki ${ }^{2}$ \\ ${ }^{1}$ Department of Respiratory Medicine, Tokyo Medical University Ibaraki Medical Center, Ibaraki, Japan \\ ${ }^{2}$ Division of Respiratory Medicine, Tokai University Hachioji Hospital, Tokyo, Japan \\ ${ }^{*}$ Corresponding Author: Hidehiro Watanabe, Department of Respiratory Medicine, Tokyo Medical University Ibaraki Medical Center, 3-20-1 Chuou, Ami, Inashiki, Ibaraki 300-0395 \\ Japan. Tel:+81-298871161, E-mail: hw-nabe4@tokyo-med.ac.jp
}

Received: February 12, 2014; Revised: March 23, 2014; Accepted: April 3, 2014

Introduction: Tracheobronchial foreign body may often be treated as asthma, chronic bronchitis or etc. especially in patients with no memories of aspiration episodes.

CasePresentation:A74-year-old woman,sufferingfrompersistentcough, wastemporarilymisdiagnosed withallergic bronchopulmonary aspergillosis and treated for six months. During this period, computed tomography (CT) findings changed from thickened bronchial walls and a "tree-in-bud" pattern to clubbing bronchiectasis and atelectasis, and no significant bacteria was detected. Finally, a vegetable core was subsequently extracted via flexible bronchofiberscopy. Although the patient's symptoms improved dramatically, the bronchopulmonary lesion remained practically.

Conclusions: We assume that chronologic CT findings of the bronchopulmonary damage by aspiration of a vegetable core, without significant detection of bacteria during the course, will be quite valuable for clinicians.

Keywords:Granuloma, Foreign-Body; Vegetables; Bronchiectasis

\section{Introduction}

Tracheobronchial foreign body (TFB) aspiration occasionally occurs in both children and adults $(1,2)$. However, this is often misdiagnosed as asthma, chronic bronchitis, or recurrent pneumonia, in patients with no alleged recollection of aspiration $(3,4)$.

Especially, in the case of vegetable containing foods, TFB is not found through a chest radiograph. As a result, a lengthy period is often required before a correct diagnosis of TFB aspiration (5). On computed tomography (CT) scan, TFBs are often difficult to detect depending on their sizes and natures, but secondary bronchopulmonary changes are sometimes visible, suggesting the presence of TFBs. Herein, we reported a patient with tracheobronchial aspiration of a vegetable core, initially diagnosed as having allergic bronchopulmonary aspergillosis (ABPA), based on asthmatic symptoms and characteristic bronchiectatic changes on a CT scan. The initial chest CT findings indicated thickened bronchial walls in the lower respiratory tract and mucus plugging, resulting in a "tree-in-bud" pattern (6), and lacked findings of TFB. Thereafter, findings changed to clubbing bronchiectasis and pulmonary atelectasis, because of mucoid impaction and deterioration. However, the patient's symptoms improved dramatically after TFB extraction via flexible bronchofiberscopy (FBSC) (7). In addition, the clubbing bronchiectasis also partially improved. We hardly found a report on observing the bronchopulmonary changes by TFB as a vegetable core by CT for six months. Moreover, during this period, no significant bacterium was detected. We considered that series of bronchopulmonary damages findings observed by CT were quite valuable.

\section{Case Presentation}

A 74-year-old Japanese woman, suffering from nonproductive cough for one month, referred to our hospital (a 500-bed city teaching hospital, serving about 560,000 people in southwest Tokyo, Japan) on April 14,2010 , due to having fever for one week. The patient had been medically treated with a calcium blocker and alendronate sodium hydrate because of hypertension

Implication for health policy/practice/research/medical education:

Involving a small foreign body composed of vegetable matter, discovery is difficult when there is no patient recollection of aspiration. We assume that series of bronchopulmonary damage findings observed by CT such as this case were quite valuable and instructive for clinicians. FBSC or virtual navigation should be actively performed.

Copyright (c) 2014, Iranian Red Crescent Medical Journal; Published by Kowsar Corp. This is an open-access article distributed under the terms of the Creative Commons Attribution License, which permits unrestricted use, distribution, and reproduction in any medium, provided the original work is properly cited. 
and osteoporosis for three years. At the time of the visit for cough, although the patient had neither sputum nor wheezing, there was evidence of only slight infiltration at the lower right lung on the chest X-ray. The patient was almost in the normal range for the ratio of forced expiratory volume in one second (FEV1: 1.42 L) to forced vital capacity (FVC: $1.88 \mathrm{~L}$ ): the lung function test result was $75.5 \%$, and only normal flora were detected in a provocation sputum culture. She was prescribed a cough medicine and an antibacterial agent (garenoxacin [GRNX], $400 \mathrm{mg} /$ day for five days) with diagnosis of bronchitis; although her cough and other clinical symptoms had reduced for several days, they subsequently returned to their original levels. She referred to our hospital again on May 18 (one month after the first consultation). On the reconsultation day, the patient's body temperature was $37^{\circ} \mathrm{C}$ and her unproductive cough was worsened. There was no evidence of swollen lymph nodes, eczema or other abnormalities. The patient's white blood cell (WBC) count was $11000 / \mu \mathrm{L}$, the percentages of neutrophils and eosinophils were $69.3 \%$ and $2.7 \%$, respectively, and C-reactive protein (CRP) concentration was $1.25 \mathrm{mg} / \mathrm{dL}$ (Table 1). No other abnormalities were observed in the blood examination. However, a chest CT showed evidence of thickened bronchial walls and mucus plugging, causing a "tree-in-bud" pattern on the right lower lobe (Figure 1A). At that time, we considered the patient temporarily diagnosed with a bronchial hypersensitivity, such as ABPA, that the imaging findings suggested. However, her unproductive cough remained dry, and no significant bacteria or fungus was detected in cultures of provocation sputum and gastric juice. In addition, both Aspergillus antigen and IgE antibody were negative, and a T-SPOT.TB test was also negative. The patient was treated with cough medicine, an inhaler (budesonid/formoterol), and a leukotriene antagonist after a short-term GRNX prescription $(400 \mathrm{mg} /$ day for five days). Although there was no episode of fever, the patient's unproductive cough was maintained, and the CT findings continued to change from thickened bronchial walls to increased clubbing bronchiectasis and mucoid impaction (Figure 1B). Treatment with a macrolide (clarithromycin [CAM], $400 \mathrm{mg} /$ day) was then added, after the patient's unproductive cough became productive in September 30. Meanwhile, there were no eosinophils in the provocation sputum, and no significant bacteria or fungi were detected in the accompanying cultures. These findings suggested that the patient was suffering from neither bronchial asthma nor ABPA. Her clinical symptoms maintained with scant improvement, and a high fever of $39^{\circ} \mathrm{C}$ and right chest pain appeared under productive cough and sputum on November 18. The patient was admitted to our hospital for pneumonopleuritis at that time with a respiratory rate of 20 breaths $/ \mathrm{min}$, blood pressure of $142 / 88 \mathrm{mmHg}$, regular pulse of 96 beats/min, and an oxygen saturation of $95 \%$ on room air. The patient's WBC count was $22500 / \mu \mathrm{L}$, the neutrophils and eosinophils percentages were $82.0 \%$ and $0.0 \%$, respectively, and CRP was at $26.29 \mathrm{mg} / \mathrm{dL}$. Since a strong inflammatory response was observed, we considered that the patient had bacteremia. The results of other blood examinations were also in the normal ranges, including the transaminases, except for lactate dehydrogenase (LDH), which was slightly elevated (243 U/L) (Table 1). Both pneumococcal and legionella urine antigen rapid tests were also negative, and no pathogenic organism was detected, although hemoculture, sputum culture, and urinary culture were performed along with antibacterial agent (cefpirome sulfate [CPR], 4 g/day, drip infusion) preinitiation treatment. Four days later, after treatment with CPR, the patient's fever abated, although a part of her productive cough continued, and WBC and CRP results improved to $12700 / \mu \mathrm{L}$ and 8.78 $\mathrm{mg} / \mathrm{dL}$, respectively. On November 25 , we considered that FBSC should be performed, as the clubbing bronchiectasis and pulmonary atelectasis at the right lower lung had not improvements (Figure 1C). FBSC findings of rubor and swelling of the bronchial mucosa at the distal intermediate bronchus were observed. When the purulent sputum was absorbed carefully, a granuloma-like structure was observed, surrounding a white foreign body at the right B9 bronchus (Figure $2 \mathrm{C}$ ), and a roughly $8 \times 5 \times 2 \mathrm{~mm}^{3}$ white foreign body was then extracted from that location (Figure 2D). As a result of a pathology search, this white foreign body was identified as a vegetable core (Figure 2B), along with a bronchus necrotic tissue. In the culture of the bronchial lavage, no significant bacteria were detected, except for scant alpha streptococcus. After extraction of the foreign body, the productive cough and sputum, as well as the clinical symptoms and blood examination results improved dramatically, and the pulmonary atelectasis also improved (Figure 1D). The patient left our hospital on November 30. The cough had disappeared completely during the 18-month period following discharge. As for the chest CT findings at May 8, 2012 (almost 18 months after the TFB extraction), the peripheral portion of the clubbing bronchiectasis observed in the right lower lung had improved, although the central portion remained (Figure $2 \mathrm{~A}$ ) .

\section{Discussion}

We reported a case of vegetable core aspiration in a healthy 74-year-old woman, presenting chronic cough and recurrent fever, who was ultimately diagnosed with TFB by FBSC, six months after her initial visit to the medical institution. The interesting point was that serial CT scans of the chest revealed various bronchopulmonary lesions, changing before and after the TFB extraction. TFB aspiration is much more prevalent in children than in adults. In a largescale pediatric report, $68 \%$ to $75 \%$ of cases occurred in children younger than $3-5$ years $(8,9)$. The TFB site was right 
Watanabe H et al.

\begin{tabular}{|c|c|c|c|c|}
\hline & \multicolumn{4}{|c|}{2010} \\
\hline & May 18 & September 30 & November 18 & November 30 \\
\hline \multicolumn{5}{|l|}{ Hematology } \\
\hline $\mathrm{WBC}, / \mathrm{mL}$ & 11000 & 7700 & 22500 & 7500 \\
\hline Seg, \% & 69.3 & 69 & 82 & 68.6 \\
\hline Eosino, \% & 2.7 & 3.2 & 0 & 3.8 \\
\hline Mono, \% & 5.6 & 7.5 & 9 & 4.4 \\
\hline Lymp, \% & 21.6 & 20.2 & 3 & 21.7 \\
\hline $\mathrm{RBC}, \times 10^{4} / \mathrm{mL}$ & 441 & 400 & 363 & 355 \\
\hline $\mathrm{Hb}, \mathrm{g} / \mathrm{dL}$ & 12.3 & 11.1 & 10.3 & 9.9 \\
\hline $\mathrm{Ht}, \%$ & 39.2 & 35.5 & 31.8 & 32.5 \\
\hline Plt,$\times 10^{4} / \mathrm{mL}$ & 41.7 & 43.2 & 76.1 & 61.5 \\
\hline \multicolumn{5}{|l|}{ Biochemistry } \\
\hline BUN, mg/dL & 11 & 15 & 10 & 12 \\
\hline $\mathrm{Cr}, \mathrm{mg} / \mathrm{dL}$ & 0.5 & 0.63 & 0.89 & 0.48 \\
\hline AST, IU/L & 22 & 14 & 28 & 15 \\
\hline ALT, IU/L & 14 & 9 & 30 & 12 \\
\hline LDH, IU/L & 155 & 180 & 243 & 138 \\
\hline Glu, mg/dL & 99 & 118 & 238 & 100 \\
\hline $\mathrm{CRP}, \mathrm{mg} / \mathrm{dL}$ & 1.25 & 0.40 & 26.29 & 0.14 \\
\hline \multicolumn{5}{|l|}{ Infection } \\
\hline Sputum Culture & NF & $\mathrm{NF}$ & NF & $\mathrm{NF}$ \\
\hline Aspergillus Ag, CI & 0.3 & ND & 0.3 & ND \\
\hline $\begin{array}{l}\text { Aspergillus Ab IgE, UA/ } \\
\mathrm{mL}\end{array}$ & $>0.34$ & ND & ND & ND \\
\hline$\beta$-D glucan, pg/mL & $>5.0$ & ND & $>5.0$ & ND \\
\hline T-SPOT.TB & $(-)$ & ND & ND & ND \\
\hline \multicolumn{5}{|l|}{ Urine antigen test } \\
\hline S.pneumoniae & ND & ND & $(-)$ & ND \\
\hline L.peumophila & ND & ND & $(-)$ & ND \\
\hline
\end{tabular}

a Abbreviations: ALT, alanine transaminase; AST, aspartate transaminase; BUN, blood urine nitrogen; CI, confidence interval; CRP, C-reactive protein; LDH, lactate dehydrogenase; NF, normal flora; ND, note done; RBC, red blood cell; WBC, white blood cell.

b Urine antigen tests (Binax NOW, Alere, California USA).

bronchus in $54.6 \%$ of cases (8) and left bronchus in $53.6 \%$ of them (9), with a nearly balanced right/left ratio. Most were discovered via image analysis (80.3\%), and roughly $20 \%$ were normal findings. Extraction by bronchoscopy under general anesthesia was effective, and most were removed in this manner $(99.72 \%)(8)$.

On the other hand, TFB aspiration is rare in adults, comprising roughly $0.33 \%$ of bronchoscopy cases (7). Ninety percent of patients have a risk factor for aspiration, with stroke being the most common of these, at 30\%. Neurological and neuromuscular diseases were involved in 58\% of all cases. TFB was suggested by medical history in $38.4 \%$. However, it was discovered by chest X-ray in only $7 \%$ of patients. The site was usually the right side (75.6\%), and the most common TFBs were animal and fish bones (39.5\%). In $90.7 \%$ of the patients, TFBs were successfully removed under FBSC, whereas in 8.1\%, TFB was extracted with a flexible bronchoscope through an endotracheal tube.

CT is useful for TFB discovery $(1,10)$. Zissin, et al. (10) examined 19 cases of TFB (11 males and 8 females, 26-89 years) and reported that CT findings in the affected lobe were volume loss, hyperlucency with air trapping, and bronchiectasis. However, as in this case, foreign bodies other than metal, teeth, dentures, and bridges are often difficult to detect by CT (7). Thus, in adults as in children, TFB is often misdiagnosed and treated as chronic bronchitis, recurrent pneumonia, persistent cough, or asthma $(3,4)$. In this case, the serial structural changes in the lung due to the foreign body were observed over time via CT. At the initial CT scan, an inflammatory reaction to the TFB impacted in the right B9 bronchus occurred, and a "tree-in-bud" pattern due to thickening of the peripheral 

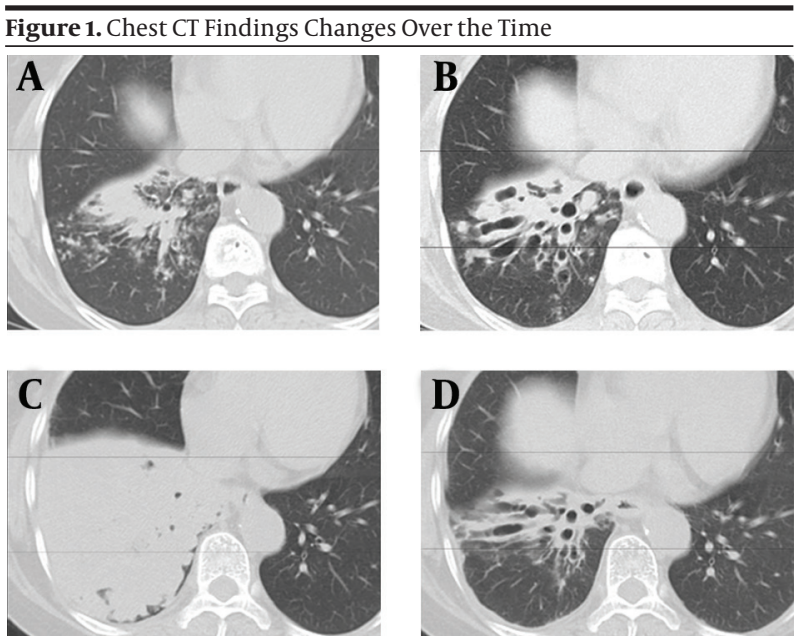

A) At May 18, 2010 (one month after the first visit), thickened bronchia walls and mucus plugging, causing a "tree-in-bud" pattern, were observed in the right lower lobe. These were considered to be from respiratory tract lesions. B) After four months (September 2), clubbing bronchiectasis was observed. C) After six months (November 18), bronchi of the right lower lobe were further dilated, and mucoid impaction was worsened, while the volume was decreased. D) One month after the TFB removal, clubbing bronchiectasis remained in the right lower lobe, although atelectasis was improved.

Figure 2. Findings of Chest CT, Bronchoscope, and Pathology of TFB
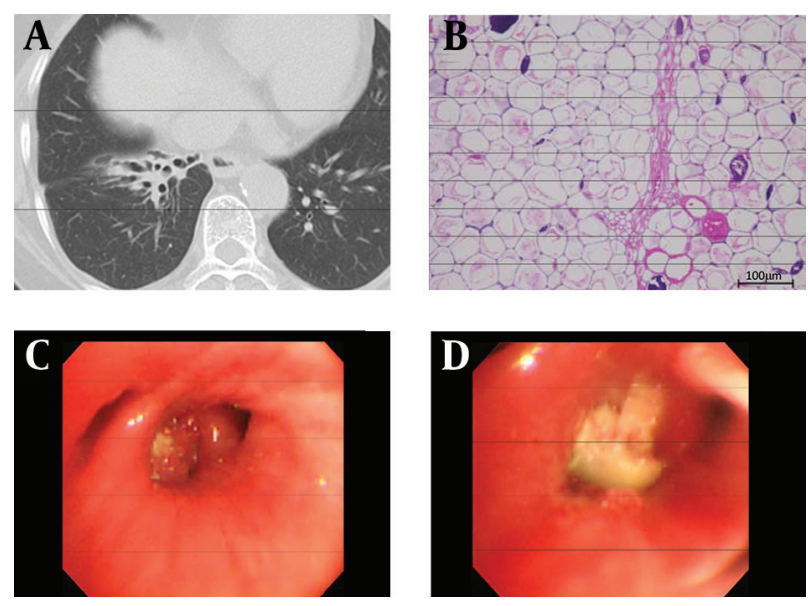

A) Almost 18 months after the TFB removal (May 8, 2012), central bronchiectasis remained in the right lower lobe; however, the check valve effect disappeared, and the peripheral area slightly improved. B) TFB pathological findings: tissue having cell walls, cytoplasm and vessels, was determined to be a hard vegetable core. (H\&E staining, magnification $\times 400$ ). C, D) Bronchoscopic findings during the extraction. C) A white TFB was observed, impacted at B9 in the right lower lobe, surrounded by granulation. D) Removal of the granulation, surrounding a white TFB, which was then extracted.

bronchial wall and mucus plugging was observed (Figure $1 A)$, resembling ABPA-like respiratory lesions (6). At this time, we suspected to ABPA, because persistent cough and asthma-like symptoms were also observed. After four months, the structure changed to clubbing bronchiectasis with mucoid impaction, suggesting that granulation was formed to cover the TFB, probably serving as a check valve (Figure 1B). After six months, when FBSC was performed, the right $\mathrm{B} 9$ bronchus and entire right lower lobar branch were occluded with granulation tissue covering the TFB (Figure 2C). A CT scan revealed that pulmonary atelectasis was developed in the lower right lobe with mucoid impaction (Figure 1C). Concomitant with this development, the clinical symptoms (hyperthermia and productive cough) worsened. However, the pulmonary atelectasis was improved by removal of the foreign body under FBSC, although the clubbing bronchiectasis remained (Figure 1D). The patient's clinical symptoms improved dramatically. In a follow-up CT almost 18 months after the removal, bronchiectasis remained central, but had improved peripherally (Figure 2A).

Involving a small foreign body composed of vegetable matter, discovery is difficult when there is no patient recollection of aspiration. In the initial phase, the TFB brought about the CT findings of "tree-in-bud" pattern. Those imaging and clinical findings caused the temporary misdiagnosis. Subsequently, it changed to mucoid impaction and bronchiectasis. We considered that series of bronchopulmonary damage findings observed by CT, such as this case, can be quite valuable and instructive for clinicians. Even if significant bacteria cannot be detected, it is important to keep track of changes in CT images in the cases of treatment-resistant persistent coughs. If mucoid impaction or bronchiectasis is observed by CT, FBSC or virtual navigation should be actively performed.

\section{Acknowledgements}

There was no acknowledgment.

\section{Authors' Contributions}

Hidehiro Watanabe designed the study, analyzed the data and wrote the paper. Tomonori Uruma and Gen Tazaki contributed to the study design.

\section{Financial Disclosure}

There was no financial interest to disclose.

\section{Funding/Support}

There was no funding or support.

\section{References}

1. Jaswal A, Jana U, Maiti PK. Tracheo-bronchial foreign bodies: a retrospective study and review of literature. Indian J Otolaryngol Head Neck Surg. 2014;66(Suppl 1):156-60.

2. Boyd M, Chatterjee A, Chiles C, Chin RJ. Tracheobronchial foreign body aspiration in adults. South Med J. 2009;102(2):171-4.

3. Deshpande KS, Haramati LB, Aldrich TK, Edelman M, Villanueva E. Foreign-body aspiration, asthma and chronic bronchitis. Am Fam Physician. 2000;62(7):1513-1517, 1519.

4. Kam JC, Doraiswamy V, Dieguez JF, Dabu J, Cholankeril M, Govind $\mathrm{M}$, et al. Foreign body aspiration presenting with asthma-like symptoms. Case Rep Med. 2013;2013:317104.

5. Nemoto K, Itou M, Senba S, Ishida J, Shimizutani N, Adachi H, et al. [A case of chronic cough and recurrent pneumonia due to 


\section{Watanabe H et al.}

an intrabronchial foreign body (fish bone), undiagnosed for 20 years].JJpn Soc Respir Endscopy. 2010;32(3):269-73.

6. Greenberger PA. Chapter 18: Allergic bronchopulmonary aspergillosis. Allergy Asthma Proc. 2012;33 Suppl 1:S61-3.

7. Mise K, Jurcev Savicevic A, Pavlov N, Jankovic S. Removal of tracheobronchial foreign bodies in adults using flexible bronchoscopy: experience 1995-2006. Surg Endosc. 2009;23(6):1360-4.

8. Zhijun C, Fugao Z, Niankai Z, Jinging C. Therapeutic experience from 1428 patients with pediatric tracheobronchial foreign body.J Pediatr Surg. 2008;43(4):718-21.

9. Korlacki W, Korecka K, Dzielicki J. Foreign body aspiration in children: diagnostic and therapeutic role of bronchoscopy. Pediatr Surg Int. 2011;27(8):833-7.

10. Zissin R, Shapiro-Feinberg M, Rozenman J, Apter S, Smorjik J, Hertz M. CT findings of the chest in adults with aspirated foreign bodies. Eur Radiol. 2001;11(4):606-11. 